\title{
Calibration of Vissim for Bus Rapid Transit Systems in Beijing Using GPS Data
}

\author{
Liu Yu, Beijing Jiaotong University \\ Lei Yu, Texas Southern University and Beijing Jiaotong University \\ Xumei Chen, Beijing Jiaotong University \\ Tao Wan, Beijing Jiaotong University \\ Jifu Guo, Beijing Transportation Research Center
}

\begin{abstract}
Bus Rapid Transit systems have grown in popularity in recent years. With the rapid development of computer technologies, using microscopic simulation models to study various strategies on planning, implementation and operation of BRT systems has become a hot research area in the field of public transportation. To make the simulation models accurately replicate field traffic conditions, model calibration is crucial. This paper presents an approach for calibrating the microscopic traffic simulation model VISSIM using GPS data for application to Beijing BRT systems. The Sum of Squared Error (SSE) of the collected versus simulated vehicle speeds at the cross-sections along the test route is specified as the evaluation index. A Genetic Algorithm is adopted as the optimization tool to minimize the SSE. Taking the Beijing North-South Central Axis BRT Corridor as a case study, it shows that the proposed approach is a practical and effective method for the model calibration.
\end{abstract}




\section{Introduction}

Bus Rapid Transit (BRT) systems have grown in popularity in recent years. With the rapid development of computer technologies, using microscopic simulation models to study various strategies on planning, implementation and operation of BRT systems has become a hot research area in the field of public transportation, particularly in cases where field experiments are difficult or expensive to conduct.

There are plenty of available microscopic simulation models used worldwide, such as VISSIM, CORSIM, PARAMICS, etc. In such models, there are a number of parameters describing the traffic flow characteristics, driving behavior, and traffic system operations, which have significant effects on simulation results. Although these models provide a set of default values for each parameter and users can conduct a simulation without calibrating them, the default values may not always be representative of the traffic situation under study. For example, the driving behavior of BRT vehicles on the exclusive lanes may be different from those on urban streets or freeways because BRT has some unique traffic characteristics (e.g., dispatching according to schedule, stopping at bus stops for serving passengers, etc.). Even BRT systems in different countries or different cities may have different characteristics. For a simulation study of BRT systems, adequate calibration based on observed traffic conditions can result in accurate and reliable simulation results, which can help transit operators make more appropriate decisions for BRT planning and implementation. So, when using a simulation model for different geographic and traffic conditions, the most important and difficult step is the calibration and validation of the model. The calibration is the process by which the values of a simulation model input parameters are refined and adjusted so that the model accurately replicates field-measured and observed traffic conditions.

The aim of this paper is to propose an approach for the automatic calibration of the driving behavior parameters of VISSIM using GPS data for application to Beijing BRT systems. A Genetic Algorithm (GA) is used for finding the best combination of VISSIM driving behavior parameters, and a particular computer simulation program named AUTOSIM is designed to run the VISSIM simulation automatically and consecutively. The validity of the proposed approach was demonstrated via a case study for the Beijing North-South Central Axis BRT Corridor. The results show that it is a practical and efficient approach for the calibration of VISSIM. 


\section{Review of Calibration Methodologies}

The problem of model calibration is very complex because of the absence of a clear analytical formulation for model users to follow. In recent years, more and more transportation researchers have realized the importance of model calibration and made great efforts to develop various methodologies to calibrate traffic simulation models.

In earlier studies, manual changes were used for calibrating model parameters (Daigle et al. 1998), which was found not efficient and practical. Fellendorf and Vortisch (2001) calibrated the car following behavior of VISSIM with measurement on the level of single vehicles, i.e., data about headways, perception thresholds, and driving characteristics. However, it is difficult for model users to collect some of such data in the field. Merritt (2003) proposed a methodology for the calibration and validation of CORSIM using empirical data. He found that extensive field data need to be collected to improve accuracy of the model calibration.

With the recent applications of ITS technologies and computational resources, there are more opportunities to calibrate simulation models based on optimization theories and algorithms. Ben-Akiva et al. (2004) presented a framework for the calibration of microscopic traffic simulation models using aggregate data. They adopted a systematic search approach based on Box's Complex algorithm for calibration, which did not require calculations of derivatives of the objective function. Nevertheless, their study found that efficient algorithms are still required to perform the calibration step. Some other algorithms, such as sequential simplex algorithm (Kim 2003) and simulated annealing algorithm (Wieland 2004), also have been studied by several researches.

In recent years, microscopic traffic simulation models have been widely used as an important tool for the analysis and design of transportation systems in China. However, many users conduct simulations simply with the default parameters provided by the model without calibrating them. The study on the calibration of traffic simulation models in China is also scarce. Sun and Yang (2004) proposed a procedure for microscopic simulation model calibration in China. They designed the experiment by using Latin Square algorithm and calibrated four of the driving behavior parameters of VISSIM, including waiting time before diffusion, minimum headway, observed vehicles, and average standstill distance. However, it takes much time to finish all the simulation experiments and these four parameters cannot represent the whole set of driving behavior parameters of VISSIM. 


\section{Proposed Calibration Approach}

\section{Identification of Calibration Parameters in VISSIM}

VISSIM is a microscopic, time-step and behavior-based simulation model developed to model urban traffic and public transit operations. It provides significant enhancements in terms of driver behavior, multi-modal transit operations, interface with planning/forecasting models, and 3-D simulation. VISSIM contains a psycho-physical car-following model for longitudinal vehicle movement and a rule-based algorithm for lateral movements. Ten calibration parameters are selected in VISSIM, including:

- Waiting Time before Diffusion - It defines the maximum amount of time a vehicle can wait at the emergency stop position waiting for a gap to change lanes in order to stay on its route. When this time is reached the vehicle is taken out of the network (diffusion) and a warning message will be written to the error file denoting the time and location of the removal.

- Minimum Headway (front/rear)-defines the minimum distance to the vehicle in front that must be available for a lane change in standstill condition.

- Maximum Deceleration - the fastest a vehicle can slow down or stop.

- -1 per Distance-used to reduce the maximum deceleration with increasing distance to the emergency stop position.

- Accepted Deceleration - the value of it is smaller than maximum deceleration but bigger than minimum deceleration, and the vehicle can slow down safely without any dangerous with accepted deceleration.

- Maximum Look Ahead Distance - the maximum distance that a vehicle can see forward in order to react to other vehicles either in front or to the side of it (within the same link). This value relates to human's physical observation ability.

- Average Standstill Distance-defines the average desired distance between stopped cars and also between cars and stop lines (signal heads, priority rules, etc.)

- Additive Part of Desired Safety Distance-this parameter and the next one (i.e. Multiple Part of Desired Safety Distance) contained with the car following model determine the saturation flow rate for VISSIM. The saturation flow rate defines the number of vehicles that can free flow through a VISSIM model during one hour. 
- Multiple Part of Desired Safety Distance-described above.

- Distance of Standing at $50 \mathrm{~km} / \mathrm{h}$ - the safety distance between two parallel cars at both the condition of stop and moving.

As the parameters mentioned above directly affect the vehicle interaction and thus can cause substantial differences in simulation results, calibration of these parameters become very important. To this end, a scientific approach is needed to calibrate these parameters.

\section{Selection of an Optimization Algorithm}

For calibration of a traffic simulation model, the difficulty is to select the best combination of the parameters being calibrated. However, all of these parameters need to be calibrated simultaneously, and each may have a different value range, which make the calibration process very complicated and time consuming. So, to identify the best parameter set for the model, an optimization algorithm is required.

A Genetic Algorithm (GA) is a search technique used in computer science to find approximate solutions to optimization and search problems. It is a particular class of evolutionary algorithms that use techniques inspired by evolutionary biology, such as inheritance, mutation, natural selection, and recombination (or crossover). It models each possible parameter set as a separate chromosome, and each chromosome is evaluated by a fitness function that represents how well it fits a given problem (Kim 2001). GA is considered robust because it performs a search from multiple points instead of starting the search at a single point. So, using the GA approach can considerably reduce the number of search steps needed and the amount of time required to complete the search when the search space is large and complex.

\section{Index of Simulation Accuracy}

To evaluate the quality of the simulation in the calibration, an evaluation index needs to be defined. There are various indexes that can be used, such as traffic volumes, average travel time, average travel speed, queue lengths, etc. This paper uses the Sum of Squared Error (SSE) between the vehicle speeds collected and those simulated at pre-defined cross-sections at a 20-meter interval along the test route, which is calculated by the following equation:

$$
S S E=\sum_{i=1}^{n}\left(v_{i}^{c}-v_{i}^{S}\right)^{2}
$$


where:

$i$ cross-section number at a 20-meter interval along the route where the speed is collected

$n$ number of cross-sections

$v_{i}^{c}$ vehicle speed collected at cross-section $i$ by GPS, and

$v_{i}^{s}$ vehicle speed simulated at cross-section $i$ by VISSIM

The speeds of vehicles in the simulation network are a good reflection of driving behavior parameters, provided the traffic volumes are known. Further, the instantaneous speed data can easily be collected by using GPS. Therefore, using the speed to evaluate the accuracy of model calibrated is not only appropriate but also practical.

For VISSIM, SSE can be considered as a function of 10 driving behavior parameters (Yu et al. 2005):

$$
S S E=f\left(x_{1}, x_{2}, x_{3}, x_{4}, x_{5}, x_{6}, x_{7}, x_{8}, x_{9}, x_{10}\right)
$$

where $x_{i}$ represents the value of the ith calibrated parameter, and $f$ is a function that is difficult to express in an analytical form. It cannot be solved through an analytical approach either. This paper establishes a simulation procedure to indirectly express the relationship between SSE and the 10 parameters.

\section{Calibration Approach Using GA}

The objective of the calibration process is to minimize the SSE, in which the GA is used as the optimization tool. The complete optimization process, which combines GA and VISSIM to find the optimal values for the 10 driving behavior parameters, consists of the following steps:

\section{Step 1. Define the Agent to Represent the Parameters}

For GA, the terms agent and gene are used. The term gene is represented by a binary digit 0 or 1 . One agent is defined as a group of genes used to represent a value of each parameter. Furthermore, one generation is defined as the specified numbers of agents. The population size is defined as the number of agents included in one generation. 


\section{Step 2. Determine the Number of Genes for Each Parameter}

For each parameter, the number of genes needed vary according to the domain of the parameter and the increment of the parameter value. The following equation is used to determine the number of genes $n_{i}$ needed for each parameter:

$$
\begin{gathered}
\max \left(x_{i}\right)-\min \left(x_{i}\right)=\alpha_{i}\left(2^{n_{i}}-1\right) \\
i=1,2,3, \ldots, 10
\end{gathered}
$$

where:

$$
\begin{array}{ll}
x_{i} & \text { the value of } i \text { th calibrated parameter } \\
\max \left(x_{i}\right) & \text { the maximum value of } x_{i} \\
\min \left(x_{i}\right) & \text { the minimum value of } x_{i} \\
\alpha_{i} & \text { the increment value of } x_{i} \text {, and } \\
n_{i} & \text { the number of genes of the agent to represent } x_{i}
\end{array}
$$

In Equation (3), $\max \left(x_{i}\right)$ and $\min \left(x_{i}\right)$ should first be identified. Then an initial value is assigned to $\alpha_{i}$ based on the number of increments desired in the search process for this parameter. Finally, $n_{i}$ is determined. After $n_{i}$ is determined, it can be substituted back into Equation (3) to calculate the final precise value of $\alpha_{i}$. The results of the calculation for all the 10 driving behavior parameters are illustrated in Table 1. The values of $\max \left(x_{i}\right)$ and $\min \left(x_{i}\right)$ are given by VISSIM.

\section{Table 1. Number of Genes and Increment of Each Parameter}

\begin{tabular}{|l|c|c|c|c|}
\hline \multicolumn{1}{|c|}{ Parameter $\boldsymbol{i}$} & $\begin{array}{c}\text { Max } \\
\left(\boldsymbol{x}_{i}\right)\end{array}$ & $\begin{array}{c}\text { Min } \\
\left(\boldsymbol{x}_{\boldsymbol{i}}\right)\end{array}$ & $\begin{array}{c}\text { \# of } \\
\text { genes } \\
\left(n_{i}\right)\end{array}$ & $\begin{array}{c}\text { Increme } \\
\boldsymbol{n t}\left(\alpha_{i}\right)\end{array}$ \\
\hline 1. Waiting time before diffusion (second) & 90 & 30 & 5 & 1.9 \\
\hline 2. Minimum headway (meter) & 1 & 0.1 & 4 & 0.06 \\
\hline 3. Maximum deceleration $\left(\mathrm{m} / \mathrm{s}^{2}\right)$ & -2 & -6 & 3 & 0.6 \\
\hline 4. -1 $\mathrm{m} / \mathrm{s}^{2}$ per distance (meter) & 150 & 50 & 5 & 1.9 \\
\hline 5. Accepted deceleration $\left(\mathrm{m} / \mathrm{s}^{2}\right)$ & -0.2 & -3 & 4 & 0.2 \\
\hline 6. Maximum look ahead distance (meter) & 300 & 200 & 4 & 6.7 \\
\hline 7. Average standstill distance (meter) & 5 & 0.2 & 3 & 0.7 \\
\hline $\begin{array}{l}\text { 8. Additive part of desired safety distance } \\
\text { (meter) }\end{array}$ & 5 & 0.2 & 3 & 0.7 \\
\hline $\begin{array}{l}\text { 9. Multiple part of desired safety distance } \\
\text { (meter) }\end{array}$ & 6 & 1 & 4 & 0.34 \\
\hline $\begin{array}{l}\text { 10. Distance of standing and at } 50 \mathrm{~km} / \mathrm{h} \\
\text { (meter) }\end{array}$ & 2 & 0.5 & 4 & 0.1 \\
\hline
\end{tabular}




\section{Step 3. Build Agent and Create Initial Generation}

Table 1 shows that a total of 39 genes are needed to represent these 10 parameters. The population size, which is the number of agents in one generation, is defined as, but not limited to 16 in this paper (the number is randomly chosen, but as a sample, it should be a relatively large number so that the better individual could be found in a very short time). In the initial generation, each gene of the agent is assigned 0 or 1 randomly.

\section{Step 4. Decode Each Agent to Parameter Value}

Equations (4) and (5) are used to decode the agent $A$ to the actual parameter value $x_{i}$.

$$
\begin{aligned}
x_{i} & =\alpha_{i} * A * B+\beta \\
A & =\left(a_{1}, a_{2}, a_{3}, \ldots, a_{n_{i}}\right) \\
B & =\left(\begin{array}{c}
2^{n_{i}-1} \\
\cdot \\
\cdot \\
2 \\
1
\end{array}\right) \\
i & =1,2,3, \ldots, 10
\end{aligned}
$$

where:

$$
\begin{array}{ll}
x_{i} & \text { the value of ith calibrated parameter, } \\
\alpha_{i} & \text { the increment value of } x_{i^{\prime}} \\
n_{i} & \text { the number of genes of the agent to represent } x_{i^{\prime}} \\
\beta_{i} & \min \left(x_{i}\right), \text { listed in Table } 1, \\
A & \text { vector to represent agent, } \\
B & \text { coefficient vector, and } \\
\alpha_{1}, \alpha_{2}, \alpha_{3}, \ldots, \alpha_{n_{i}} & 0 \text { or } 1
\end{array}
$$




\section{Step 5. AUTOSIM}

Fitness is used to evaluate the quality of the agent. The higher the fitness, the better the agent. In other words, the set of 10 driving behavior parameters is better if the fitness resulted from the simulation is higher. In the proposed approach, SSE is used as the fitness function, where the fitness is the highest when SSE is the minimum. As mentioned earlier, $f$ is a function that cannot be expressed in an analytical form. As such, a simulation procedure named AUTOSIM is designed to express the relationship between SSE and the 10 parameters. This procedure automatically runs VISSIM with different values of the input parameters and generates the outputs of SSE. The flowchart of the AUTOSIM procedure, programmed with Visual Basic 6.0, is shown in Figure 1.

\section{Figure 1. The Flowchart of AUTOSIM}

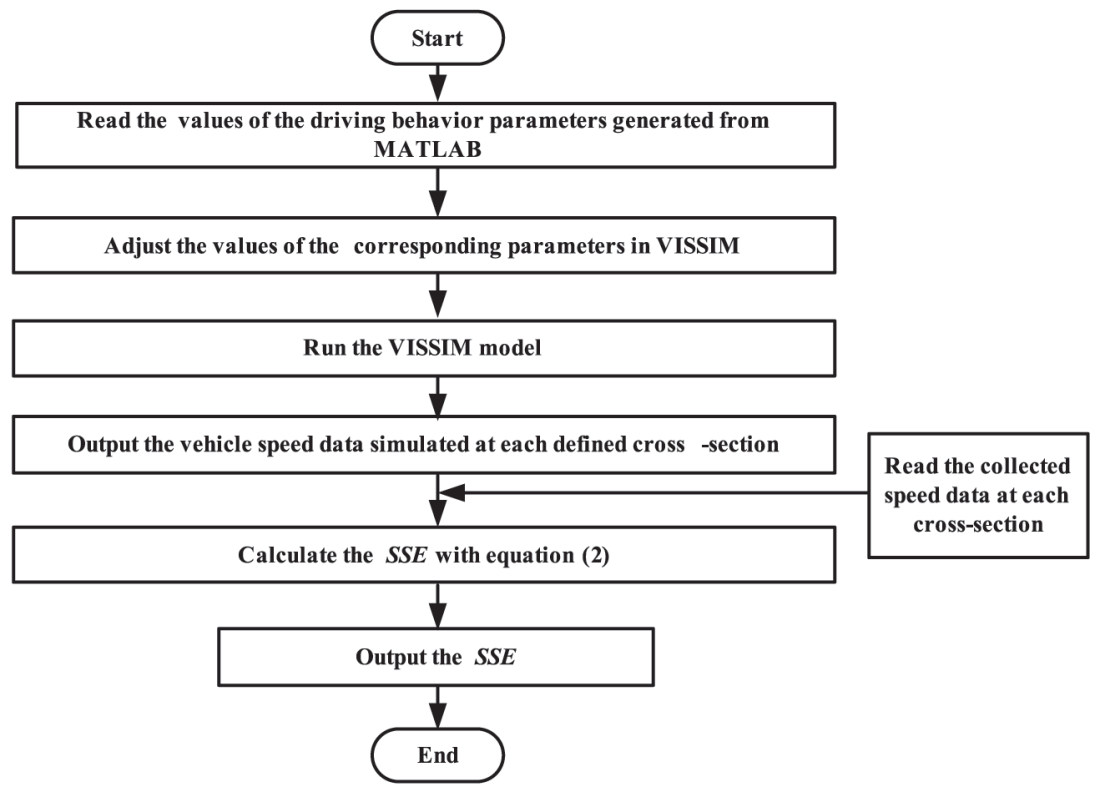

\section{Step 6. Evaluate the Fitness of Agents and Select the Best Agent}

With the AUTOSIM, the evaluation of each agent in one generation can be performed. Before the AUTOSIM is entered, Equations (4) and (5) are used to decode 
the agent to derive the values of the parameters. After all agents in the current generation have gone through the AUTOSIM, the fitness of agents is evaluated based on SSE, and then the best agent is selected. If the SSE of the best agent in the current generation does not satisfy a pre-defined criterion, the following step is taken to create the next generation of agents. Otherwise, the process stops, and the optimal parameter values are derived.

\section{Step 7. Select, Crossover and Mutate Agent}

In GA, select, crossover and mutate are three chief operators needed in creating the next generation of agents. Selection is based on the probability, and the agents with higher fitness values will most likely be selected. To crossover, two agents interchange part of their genes to create two new agents. One agent is mutated to create a new agent by changing one of its genes from 1 to 0 or from 0 to 1 .

\section{Step 8. Create a New Generation}

The search for the optimum values is an iterative process. After the operators of selection, crossover and mutation are carried out to the agents of the former generation, more agents will be produced to form a new generation while keeping the same population size.

\section{Step 9. Implementation of the approach}

The MATLAB platform is used for programming to implement the GA-based approach. The Genetic Algorithm Toolbox developed by University of Sheffield also is used. This toolbox provides functions to implement the operators of selection, crossover and mutation. The final program integrates the MATLAB, GA toolbox, Visual Basic, and VISSIM.

\section{Case Study for Beijing BRT Systems}

Beijing is the capital and most congested city in China, with the number of registered motor vehicles exceeding 2 million (including 1.28 million cars). Traffic speed on some urban roads averaged $12 \mathrm{~km} / \mathrm{h}$ in 2003, compared to $20 \mathrm{~km} / \mathrm{h}$ in 1996 , and $45 \mathrm{~km} / \mathrm{h}$ in 1994. More than 40 percent of residents spent more than one hour getting to work, and 87 percent of road sections are constantly congested. Therefore, traffic congestion will be a major challenge for Beijing for the 2008 summer Olympic Games. Developing BRT is, of course, one of the feasible solutions for Beijing. 
With technical support from the Beijing Energy Foundation, the Beijing NorthSouth Central Axis BRT Corridor was launched in December 2004 as a test BRT corridor, which was the first closed BRT system (stations requiring fare collection before boarding) in China and only the second outside Latin America. The Beijing North-South Central Axis BRT Corridor is 15.8 kilometers long and passes the Qianmen commercial area and four ring roads. This BRT Corridor was completed in two phases. Phase I opened for operation on December 25, 2004, and starts at Qianmen toward Muxiyuan, with 5 stops, and is 5 kilometers long. Phase ll opened on December 30, 2005, and goes from Muxiyuan to Demaozhuang, with 11 stops, and is 11 kilometers long.

\section{Test Site}

The test site in this paper is the Phase I of the Beijing North-South Central Axis BRT Corridor, which is $5 \mathrm{~km}$ long, with $2.5 \mathrm{~km}$ exclusive bus lanes and $2.5 \mathrm{~km}$ mixed use roadway, including eight intersections. The exclusive bus lanes are separated from cars at the center of the road, and the bus stops are $5 \mathrm{~m}$ wide and 40-60 m long. The BRT buses operating on the exclusive lanes are 18 meters long, air-conditioned, with left-open doors, low floors and a capacity of 200 passengers. The BRT buses run from 5:00 am to 10:30 pm, with a headway of 2-3 minutes at peak hour and 4-5 minutes at off-peak hour. The layout of the test site is shown in Figure 2.

\section{Figure 2. Layout of the Test Site}

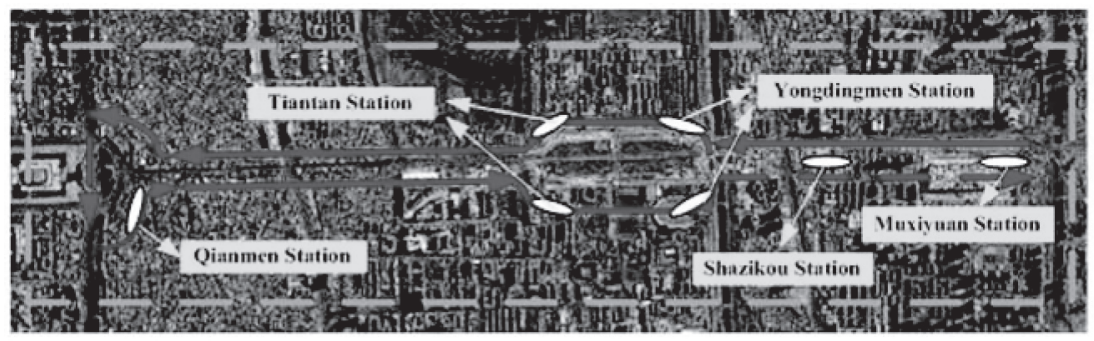

\section{Data Collection}

For the purpose of calibration, traffic and GPS data need to be collected. Traffic data include the traffic volumes entering into the network, the turning ratio at each intersection, the signal timing of the signalized intersections, the schedule 
of the BRT operation, and the BRT dwelling time at each bus stop. GPS data are instantaneous speed of vehicles collected by using GPS equipped on the test BRT vehicle or car. The vehicle equipped with GPS is driven along the test route in a similar way to the floating car method. In this study, both the speeds of BRT vehicle and car were collected. Ten repeated cycle runs of the test were conducted along the same route.

Six sections for BRT and two sections for car were defined to conduct the data collection, as shown in Figure 3 and Figure 4.

\section{Figure 3. Six Sections for BRT along the North-South Central Axis BRT Corridor}

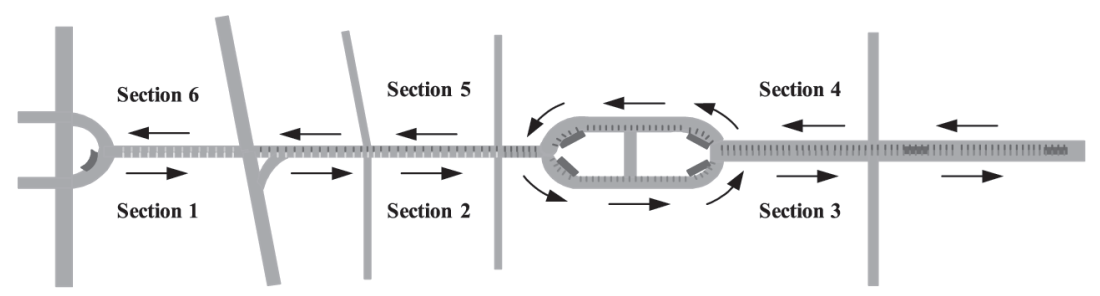

\section{Figure 4. Two Sections for Car around the North-South Central Axis Road}

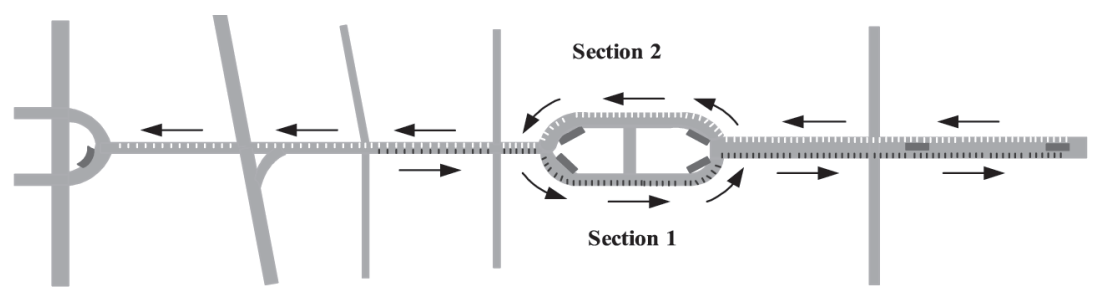

Table 2 describes the sections defined.

To calculate the SSE of each section, the simulated speeds of vehicles should be output by setting up detectors along the test route in VISSIM. 
Table 2. Description of the Sections Defined

\begin{tabular}{|c|c|c|c|}
\hline $\begin{array}{c}\text { Vehicle } \\
\text { Type }\end{array}$ & $\begin{array}{c}\text { Section } \\
\text { Number }\end{array}$ & Length $(\boldsymbol{m})$ & Route Direction \\
\hline \multirow{4}{*}{ BRT } & 1 & 820 & Qianmen to Zhushikou \\
\cline { 2 - 4 } & 2 & 1150 & Zhushikou to Tiantan West Gate \\
\cline { 2 - 4 } & 3 & 2360 & Tiantan West Gate to Muxiyuan \\
\cline { 2 - 4 } & 4 & 2360 & Muxiyuan to Tiantan West Gate \\
\cline { 2 - 4 } & 5 & 1150 & Tiantan West Gate to Zhushikou \\
\hline \multirow{4}{*}{ Car } & 6 & 820 & Zhushikou to Qianmen \\
\cline { 2 - 4 } & I & 2980 & Tianqiao to Muxiyuan \\
\hline
\end{tabular}

\section{Model Calibration and Results}

Because of the difference between BRT and general traffic in operation, the values of driving behavior parameters of BRT vehicles may be different from those on urban streets or freeways. Therefore, both the driving behavior parameters of $B R T$ vehicles and cars should be calibrated. To carry out the calibration process, the MATLAB platform is used for programming to implement the calibration approach and the Genetic Algorithm Toolbox in MATLAB is used for performing the GA operation. In this MATLAB platform, the AUTOSIM program mentioned earlier can be called to run the VISSIM model automatically.

In this case study, a criterion is specified on when the program should stop. The stopping criterion is specified as when either 10 consecutive generations have the same SSE, or the difference between SSEs from two consecutive runs is less than or equal to 1 percent (not including two consecutive generations with the same SSE). Table 3 illustrates the results from the calibration, in which the criterion is met after the program runs for 28 generations for BRT and 25 generations for Cars.

Tables 4 and 5 show part of the results of SSE from the program for BRT and cars. 
Table 3. The Values of Default and Optimal Parameters

\begin{tabular}{|c|c|c|c|}
\hline \multirow{2}{*}{ Driving Behavior Parameter } & \multirow{2}{*}{$\begin{array}{c}\text { Default } \\
\text { Value }\end{array}$} & \multicolumn{2}{|c|}{ Optimal Value } \\
\hline & & $B R T$ & Car \\
\hline Waiting time before diffusion $(s)$ & 60.00 & 43.30 & 64.20 \\
\hline Minimum headway $(m)$ & 0.50 & 0.22 & 1.00 \\
\hline Maximum deceleration $\left(\mathrm{m} / \mathrm{s}^{2}\right)$ & -4.00 & -4.40 & -4.40 \\
\hline$-1 \mathrm{~m} / \mathrm{s}^{2}$ per distance $(\mathrm{m})$ & 100.00 & 133.20 & 78.80 \\
\hline Accepted deceleration $\left(\mathrm{m} / \mathrm{s}^{2}\right)$ & -1.00 & -0.10 & -0.30 \\
\hline Maximum look ahead distance $(m)$ & 250.00 & 300.0 & 273.70 \\
\hline Average standstill distance $(\mathrm{m})$ & 2.00 & 1.60 & 1.60 \\
\hline $\begin{array}{l}\text { Additive part of desired safety distance } \\
(m)\end{array}$ & 2.00 & 1.60 & 4.40 \\
\hline $\begin{array}{l}\text { Multiple part of desired safety distance } \\
(m)\end{array}$ & 3.00 & 2.70 & 3.72 \\
\hline $\begin{array}{l}\text { Distance of standing and at } 50 \mathrm{~km} / \mathrm{h} \\
(m)\end{array}$ & 1.00 & 2.00 & 1.90 \\
\hline
\end{tabular}

Table 4. Simulation Results of SSE from 28 Iterations for BRT

\begin{tabular}{|c|c|c|c|}
\hline Generation\# & $\begin{array}{c}\text { SSE of the Best } \\
\text { Agent }\end{array}$ & $\begin{array}{c}\text { Difference of SSEs } \\
\text { with the Previous } \\
\text { Generation }\end{array}$ & \% Difference \\
\hline 0 & 3253 & & \\
\hline 5 & 3158 & 352 & 10.04 \\
\hline 10 & 2448 & 178 & 6.79 \\
\hline 15 & 1885 & 48 & 2.46 \\
\hline 20 & 1857 & 28 & 1.47 \\
\hline 28 & 1513 & 14 & 0.91 \\
\hline
\end{tabular}

Table 5. Simulation Results of SSE from 25 Iterations for Cars

\begin{tabular}{|c|c|c|c|}
\hline Generation\# & $\begin{array}{c}\text { SSE of the Best } \\
\text { Agent }\end{array}$ & $\begin{array}{c}\text { Difference of SSEs } \\
\text { with the Previous } \\
\text { Generation }\end{array}$ & \% Difference \\
\hline 0 & 1929 & & \\
\hline 5 & 2176 & 0 & 0.00 \\
\hline 10 & 1470 & 32 & 2.13 \\
\hline 15 & 1139 & 22 & 1.88 \\
\hline 20 & 1005 & 17 & 1.64 \\
\hline 25 & 852 & 8 & 0.98 \\
\hline
\end{tabular}


In Tables 4 and 5, generation number 0 means the default parameter values. For the first generation, the agents are created at random, so the SSE of the best agent in this generation is greater than the default SSE. However, in the following generations, the SSE of the latter generation is smaller than (or equal to) the former. Take BRT as an example. After the computer program runs for 5,10 and 28 generations, its values are reduced to 3158,2448 and 1513 , respectively. Thus, the SSE has decreased almost 53.5 percent when the parameters are changed from the default values to the optimal ones. To the planning of BRT projects in practical terms, this value means potential benefits, such as operating cost savings, improved service planning and level of service, because BRT operators can make better decisions according to the simulation results provided by the model calibrated, which accurately replicates the observed traffic conditions.

To visualize the results, the speeds simulated using the default parameter values, collected by using GPS system and optimized after the calibration, are compared. Figure 5 shows an example of the speed profile for BRT, and Figure 6 is an example of the speed profile for cars.

For comparison purposes, the errors between the simulated speeds and the collected speeds at cross-sections are computed. For BRT, with the default parameter values, the percentage of relative errors greater than 20 percent of the total sample is 80.3 percent, compared with only 19.7 percent when the optimal parameter values are used. Furthermore, the overall Standard Deviation with default values is 19.32 , and only 5.55 with optimal values. The results show that the accuracy of the model after calibration is improved considerably.

\section{Model Validation}

To determine that the model calibrated accurately represents the real system, the Tianqiao Intersection is used to do the model validation. The measured volumes of the South Approaches of this intersection, the simulated volumes using the default parameters, and the simulated volumes using the calibrated parameters are compared (shown in Figure 7). From this figure, it is found that the model calibrated matches the field observations within a small error range, in which the maximum relative error is only 2.5 percent. However, the maximum error of the model with default parameters can reach to 27.4 percent. 
Figure 5. Comparison of BRT Vehicle Speed Profile for Section 1

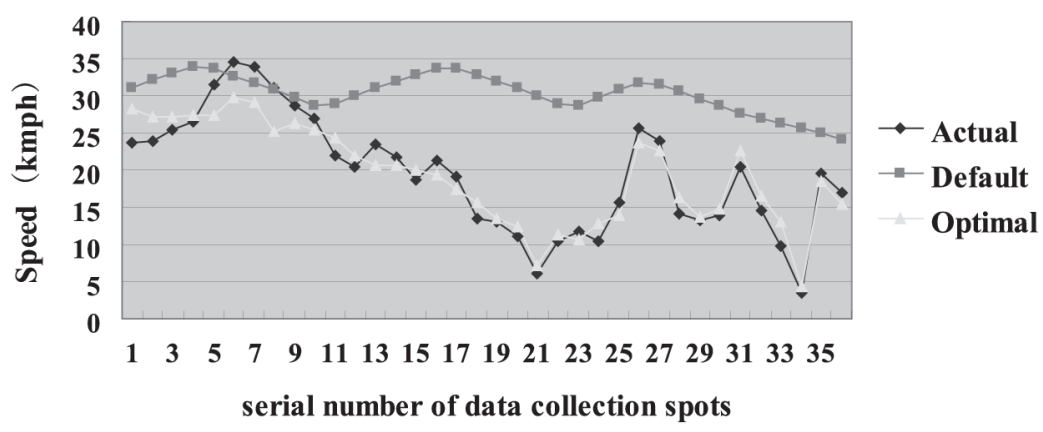

Figure 6. Comparison of Car Speed Profile for Section II

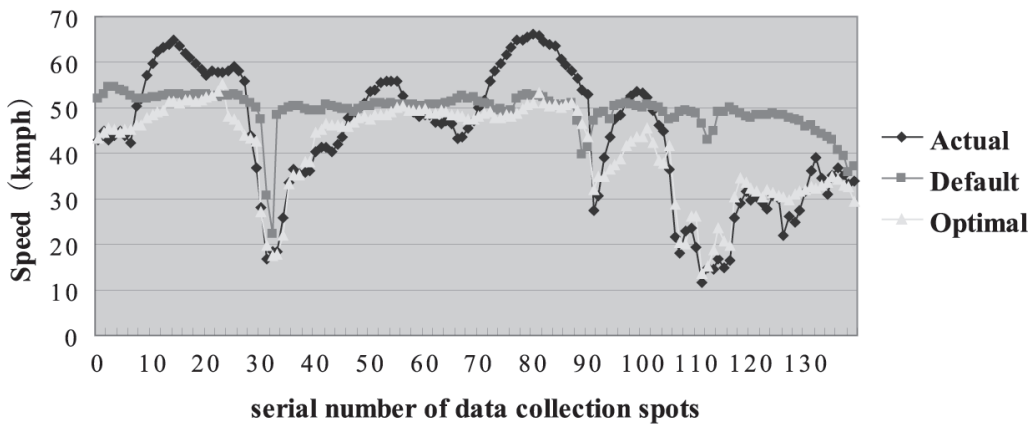

Figure 7. Comparison of Volumes with Default versus Optimal values

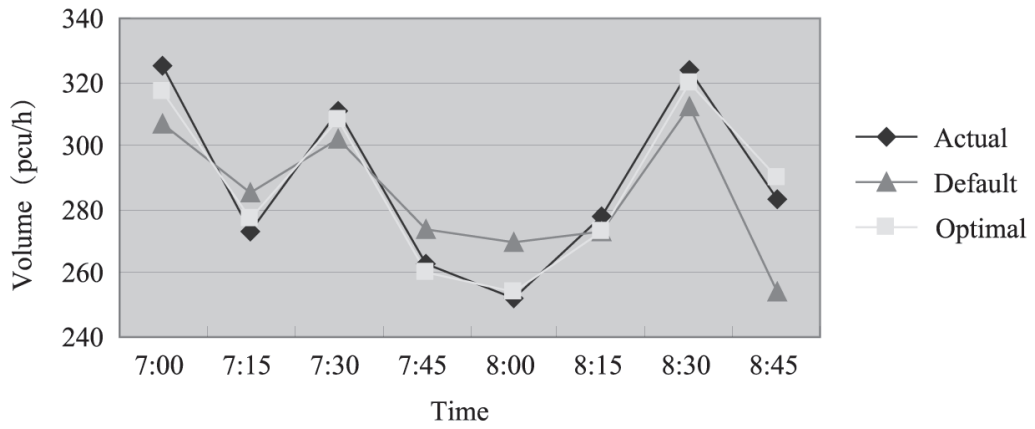




\section{Conclusions and Recommendations}

This paper presented an approach for calibrating the microscopic simulation model VISSIM for Beijing BRT systems using GPS data. The SSE of the collected versus simulated vehicle speeds at the cross-sections was defined as the evaluation index. The objective of the calibration is to search for the best combination of the parameters that minimize the SSE, and a Genetic Algorithm is adopted as an optimization tool to implement the search process. For the efficiency improvement of calibration, a computer program is developed to integrate the MATLAB, Visual Basic, and VISSIM. The validity of the proposed approach was demonstrated via a case study for the Beijing North-South Central Axis BRT Corridor. Both the field instantaneous speeds of BRT vehicle and cars along the test route were collected by using GPS for calibration. The case study shows that the proposed approach is a practical and effective method for calibrating the VISSIM model.

Since this study used only one Measure of Effectiveness (MOE), i.e., SSE, for model calibration, the performance of other MOEs is uncertain. Further research is recommended to include more MOEs (e.g., delay or queue) in the calibration process. Furthermore, with an increased trend of simulation application for study on BRT systems, other types of BRT (e.g., all arterial or all exclusive right-of-way) should be considered to verify the performance of the proposed approach. Doing so will provide more insight on the feasibility of the proposed approach.

\section{Acknowledgements}

This paper was prepared based on a project (Y0604002040691) funded by Beijing Municipal Science \& Technology Commission titled "BRT Planning, Operational and Organizational Coordination Techniques," the projects of "Talent Building" Foundation of Beijing Jiaotong University (YSJ04001), and National Natural Science Foundation of China (50208002).

\section{References}

Ben-Akiva, M.E., D. Darda, M. Jha, H.N. Koutsopoulos, and T. Toledo. 2004. Calibration of microscopic traffic simulation models with aggregate data. Proceedings of the 83rd Transportation Research Board Annual Meeting, Washington, DC. 
Daigle, G., M. Thomas, and M. Vasudevan. 1998. Field applications of CORSIM: I-40 freeway design evaluation, Oklahoma city, OK. Proceedings of the 1998 Winter Simulation Conference:1161-1167.

Fellendorf, M., and P.Vortisch. 2001. Validation of the microscopic traffic flow model VISSIM in different real-world situations. Paper presented at the 81st Transportation Research Board Annual Meeting, Washington, DC.

Kim, K., and L.R. Rilett. 2003. Simplex based calibration of traffic micro-simulation models using ITS data. Presented at 82nd Annual Meeting of the Transportation Research Board, Washington, DC.

Kim, K., and L.R. Rilett. 2001. Genetic-algorithm-based approach for calibrating microscopic simulation models. 2001 IEEE Intelligent Transportation Systems Conference Proceedings: 298-704.

Merritt, E. 2003. Calibration and validation of CORSIM for Swedish road traffic conditions. In Preprints of the 83rd Transportation Research Board Annual Meeting, Washington, DC.

Sun,J., and X. Yang, 2004. Study on calibration of microscopic traffic simulation models-Taking VISSIM as an example. COMPUTER AND COMMUNICATIONS, 3(22): \#3-6.

Wieland, F., and T.C. Holden. 2004. Model calibration by simulaiton optimization. The MITRE Corporation.

Yu, L., X. Li, and W. Zhuo. 2005. GA-Based calibration of VISSIM for Intercontinental Airport of Houston (IAH) network using GPS data. 84th Transportation Research Board Annual Meeting CD-ROM, Washington, DC.

\section{About the Authors}

LIU YU (yuliu1991@163.com) is a Ph.D. candidate at School of Traffic and Transportation, Beijing Jiaotong University in Beijing, China. Her research interests involve Bus Rapid Transit development, transportation simulation, and Intelligent Transportation Systems.

LEI Yu (yu_lx@tsu.edu) is Professor and Chairman of Department of Transportation Studies, Texas Southern University. He is also Changjiang Scholar of Beijing Jiaotong University and has managed 50 research projects and has published over 100 scientific papers. 
XUmei Chen (chenxumei@jtys.bjtu.edu.cn) is an associate professor of School of Traffic and Transportation, Beijing Jiaotong University in Beijing, China. Her research interests involve ITS technologies, network planning of urban rail transit, and industry policy of transportation.

TAO WAN (seaowner800@163.com) is a graduate research assistant at School of Traffic and Transportation, Beijing Jiaotong University in Beijing, China. His research interests involve vehicle exhaust emission testing, Bus Rapid Transit development, and transportation modelling.

Jıfu Guo (guojf@bjtrc.org.cn) is the Deputy Director of Beijing Transportation Research Centre (BTRC). His main research areas traffic forecasting, metro/highway planning, traffic impact analysis, and traffic environment analysis. 